\title{
Effect of Gate Structure on the Performance of GaN Junctionless FinFETs
}

\author{
Ki-Sik Im, Sung Jin An, Christoforos G. Theodorou, Gérard Ghibaudo, Fellow, IEEE, Sorin \\ Cristoloveanu, Fellow, IEEE, and Jung-Hee Lee, Senior Member, IEEE
}

\begin{abstract}
We investigated the performances of GaN junctionless nanochannel fin-shaped field-effect transistors (FinFETs) with two different types of gate structures; overlapped- and partially covered-gate. DC, low-frequency noise (LFN), and pulsed I-V characterization measurements were performed and analyzed together in order to identify the conduction mechanism and examine both the interface and buffer traps in the devices. The fabricated GaN junctionless device with overlapped gate structure exhibits improved DC and noise performance compared to the device with partially covered-gate, even though its gate length is much larger. The LFN behavior was found to be dominated by carrier number fluctuations (CNF). At off-state, the device with partially covered-gate exhibits generation-recombination ( $g-r)$ noise on top of $1 / f$ noise. This superposition is correlated with the severe current collapse revealed by pulsed I-V measurements. In contrast, the device with overlapped gate shows clear $1 / f$ behavior without g-r noise.
\end{abstract}

Index Terms - GaN, Junctionless, Nanochannel, FinFET, Low-frequency noise, generation-recombination noise, Current collapse.

\section{Introduction}

$\mathrm{H}$ ighly-doped Gallium nitride (GaN) nanochannel fin-shaped field-effect transistor (FinFET) without $\mathrm{AlGaN} / \mathrm{GaN}$ heterojunction was initially proposed and demonstrated by our groups [1]-[2]. The conduction mechanism of this transistor is governed by the bulk current flowing through the inside of the heavily-doped $\mathrm{GaN}$ nanochannel arrays, which is totally different from the two-dimensional electron gas (2-DEG) channel in AlGaN/GaN

Manuscript received Oct. 2019. This work was supported by the National Research Foundation of Korea (NRF) funded by the Ministry of Education, Science and Technology (MEST) (No. NRF-2018R1A6A1A03025761, NRF-2019R1I1A1A01064011). This research was partially supported by Nano-Material Technology Development Program through the NRF funded by the Ministry of Science, ICT and Future Planning (2009-0082580).

K.-S. Im is Advanced Material Research Center, Kumoh National Institute of Technology, Gumi, 39177, South Korea (e-mail: ksim@kumoh.ac.kr).

S. J. An is Department of Advanced Materials Science and Engineering, Kumoh National Institute of Technology, Gumi, 39177, South Korea.

C. G. Theodorou, G. Ghibaudo, and S. Cristoloveanu are Institute of Microelectronics, Electromagnetism, and Photonics, Grenoble Institute of Technology, 38016, Grenoble, France.

J.-H. Lee is School of Electronics Engineering, Kyungpook National University, Daegu, 41566, South Korea. heterojunction FinFETs. The GaN junctionless device exhibited outstanding device performances (high $\mathrm{I}_{\text {on }} / \mathrm{I}_{\text {off }}$ ratio, excellent subthreshold swing, and large breakdown voltage), added to its simple epitaxial growth and fabrication process [1]-[2]. The fully gate-covered nanochannel structure has been adapted to GaN-based FinFET, omega FinFET, and gate-all-around (GAA) FET because this architecture provides superior device performances, such as improved on-current and reduced current collapse owing to decreased access resistance and less back-gating effect [2]-[6].

Generally, GaN contains a large density of defects, impurities, and dislocations due to the lack of native substrate material. These defects can behave as charge trapping centers, which gradually degrade the device performance and give rise to severe reliability concerns. Low frequency noise (LFN) measurements have great merits in analyzing the interface and/or oxide traps [7] and can also help identifying the conduction mechanism in semiconductor devices. There have been many studies dedicated to $\mathrm{LFN}$ for $\mathrm{AlGaN} / \mathrm{GaN}$ MISHEMTs, AlGaN/GaN FinFETs, AlGaN/GaN omega FETs, AlInGaN/GaN Fin-HEMTs, and GAA FETs with/without $\mathrm{AlGaN} / \mathrm{GaN}$ heterojunction [8]-[11]. It is generally admitted that the origin of the noise mechanism in most of GaN-based 3-dimensional (3D) devices is mainly due to the carrier number fluctuations (CNF) [7]. However, no detailed noise investigation has been conducted regarding the conduction mechanism of $\mathrm{GaN}$ junctionless FinFET. In this work, we characterize novel GaN junctionless FinFETs with two different types of gate structures, overlapped- and partially covered-gate, by considering DC, LFN, and pulsed I-V measurements.

\section{EpItaXy Growth AND DeVICE FABRICATION}

The heavily-doped GaN layer was simply grown on a sapphire (0001) substrate by metal organic chemical vapor deposition (MOCVD). The epitaxial layers consist of 2 $\mu \mathrm{m}$-thick highly-resistive undoped $\mathrm{GaN}$ buffer layer and 150 nm-thick Si-doped n-type GaN layer which were sequentially grown on sapphire substrate. Hall effect measurements at room temperature showed a carrier density of $1 \times 10^{18} \mathrm{~cm}^{-3}$, an electron mobility of $280 \mathrm{~cm}^{2} / \mathrm{V} \cdot \mathrm{s}$, and a sheet resistance $\left(\mathrm{R}_{\mathrm{sh}}\right)$ of $1490 \Omega /$ sq. 
For the device fabrication, mesa etching for device isolation was performed using inductively coupled plasma-reactive ion etching (ICP-RIE) using a $\mathrm{Cl}_{2}$ gas. After depositing a 20 nm-thick $\mathrm{HfO}_{2}$ and 100 nm-thick $\mathrm{SiO}_{2}$ hard mask layer, a fin pattern with fin width $\left(\mathrm{W}_{\text {fin }}\right)$ of $100 \mathrm{~nm}$ along $\langle 11-20\rangle$ direction was defined by electron-beam lithography (EBL) and etched by ICP-RIE. A tetramethyl ammonium hydroxide (TMAH) wet solution $\left(5 \%\right.$ solution at $\left.90{ }^{\circ} \mathrm{C}\right)$ was applied to decrease the slope and size of the fin and to reduce the plasma damage and surface roughness [4], [12], which resulted in 36 parallel fin arrays with width $\left(\mathrm{W}_{\text {fin }}\right)$ of $50 \mathrm{~nm}$, height $\left(\mathrm{H}_{\mathrm{fin}}\right)$ of $200 \mathrm{~nm}$, and length $\left(\mathrm{L}_{\text {fin }}\right)$ of $2 \mu \mathrm{m}$. Si/Ti/Al/Ni/Au stack for ohmic contacts was then deposited by electron-beam evaporator, followed by rapid thermal annealing (RTA). Finally, Ni gate metal was deposited to form the gate length $\left(\mathrm{L}_{\mathrm{g}}\right)$ of $0.5 \mu \mathrm{m}$ and $2 \mu \mathrm{m}$ for the partially covered- and the overlapped-device, respectively. It is noticed that $\mathrm{L}_{\mathrm{g}}$ of the FinFET with overlapped-gate structure is not the length of the gate metal, but rather corresponds to the $\mathrm{L}_{\text {fin }}$ of the device. The device structure is illustrated in Fig. 1.

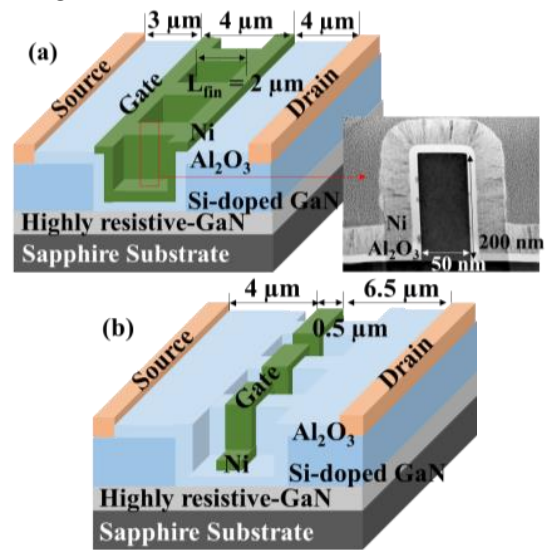

Fig. 1. Schematic illustration of the proposed GaN junctionless FinFETs (a) overlapped- and (b) partially covered-gate structure. Inset of Fig. 1(a) shows cross-sectional TEM image of the perfectly rectangular GaN nanochannel fin.

\section{CHARACTERIZATION RESULTS AND DISCUSSION}

Fig. 2 shows the drain current, transfer, and output curves of the fabricated GaN junctionless nanochannel FinFETs. Both devices show similar characteristics, such as a threshold voltage, $\mathrm{V}_{\text {th }}$, around $-0.5 \mathrm{~V}, \mathrm{I}_{\text {on }} / \mathrm{I}_{\text {off }}$ ratio of $10^{5}$, subthreshold swing of $110 \mathrm{mV} / \mathrm{dec}$, broaden transconductance $\mathrm{g}_{\mathrm{m}}$, and low saturation voltage of $\sim 3 \mathrm{~V}$. The reason for the negative $\mathrm{V}_{\text {th }}$ is the uncompleted depletion in off-state of the heavily-doped $\mathrm{GaN}$ fin channel with width of $50 \mathrm{~nm}$. The broad $\mathrm{g}_{\mathrm{m}}$ curves that extend to a gate voltage $\left(\mathrm{V}_{\mathrm{g}}\right)$ of $1.5 \mathrm{~V}$ indicate the improvement of the device linearity due to (i) the current contribution of surface accumulation channel formed at higher $\mathrm{V}_{\mathrm{g}}$ in addition to the channel current through the fin body and (ii) the small dynamic access resistance of the FinFETs [6], [13]. From the hysteresis under the double sweep of the $\mathrm{V}_{\mathrm{g}}$ in Fig. 2(a), it is clearly observed that the partially covered-device suffers from the severe trapping effect with high hysteresis of $220 \mathrm{mV}$ when compared to the value of $40 \mathrm{mV}$ for the overlapped-device. The maximum drain current $\left(\mathrm{I}_{\mathrm{d}, \mathrm{max}}\right)$ and maximum transconductance $\left(\mathrm{g}_{\mathrm{m}, \max }\right)$ are however different. The device with partially
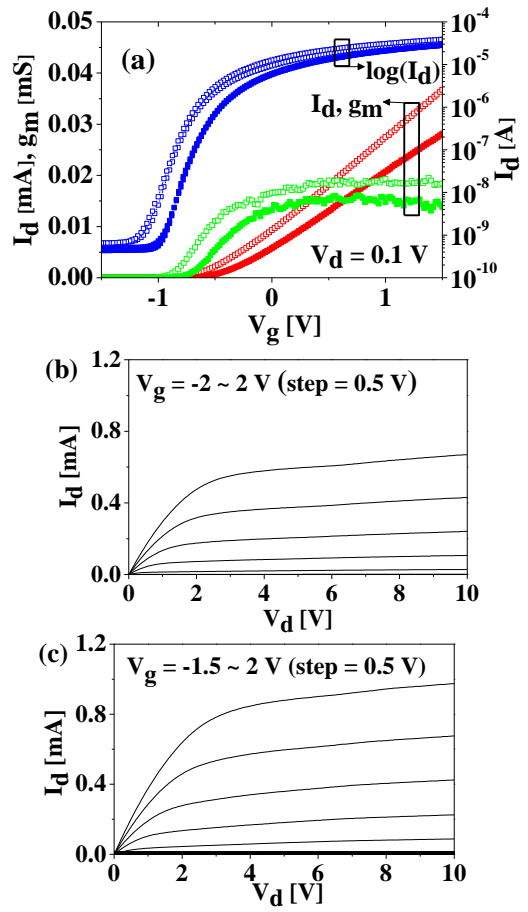

Fig. 2. (a) Transfer curves (double sweep of gate voltage) of the fabricated GaN junctionless FinFETs with overlapped- (filled rectangular) and partially covered-gate (empty rectangular) structure at $V_{d}=0.1 \mathrm{~V}$. Corresponding $I_{d}-V_{d}$ curves for devices with (b) overlapped- and (c) partially covered-gate structure.

covered-gate is shorter $\left(\mathrm{L}_{\mathrm{g}}=0.5 \mu \mathrm{m}\right)$ and exhibits higher $\mathrm{I}_{\mathrm{d}, \max }$ and $\mathrm{g}_{\mathrm{m}, \max }$ values than those of the device with the overlapped-gate and $\mathrm{L}_{\mathrm{g}}$ of $2 \mu \mathrm{m}$. Even though the $\mathrm{L}_{\mathrm{g}}$ for the device with partially covered-gate is 4-times shorter than the device with the overlapped-gate, the $\mathrm{I}_{\mathrm{d}, \max }$ for the partially covered-device exhibits only 1.4-times higher value than that of the overlapped-device due to the larger access resistance (Fig. 2(b) and Fig. 2(c)).

LFN measurements were performed at room temperature, varying the gate bias from subthreshold to strong accumulation region in the linear region at $\mathrm{V}_{\mathrm{d}}=0.1 \mathrm{~V}$. A fully automatic $\mathrm{LFN}$ measurement system from Synergie Concept is used in the frequency ranges from $4 \mathrm{~Hz}$ to $10^{4} \mathrm{~Hz}$ [13]. The drain current noise spectral densities $\left(\mathrm{S}_{\mathrm{Id}}\right)$ in Fig. 3(a) show $1 / f^{\prime}$ shape with $\gamma$ close to 1 in the entire frequency bandwidth. Regardless of the gate structure, both devices exhibit almost identical drain current noise level $\mathrm{S}_{\mathrm{Id}}$ at $\mathrm{I}_{\mathrm{d}}=0.02 \mathrm{~mA}$.

According to the CNF model, LFN originates from trapping-related fluctuations in free carrier concentration, which is mostly ascribed to the trapping/detrapping of free carriers between the interface traps and the surface channel. The CNF equation can be expressed as follows, showing $\mathrm{S}_{\mathrm{Id}} / \mathrm{I}_{\mathrm{d}}{ }^{2}$ is proportional to $\left(\mathrm{g}_{\mathrm{m}} / \mathrm{I}_{\mathrm{d}}\right)^{2}$ [7], [15],

$$
\frac{S_{I d}}{I_{d}^{2}}=\left(\frac{g_{m}}{I_{d}}\right)^{2} S_{V f b} \text { with } S_{V f b}=\frac{q^{2} k T \lambda N_{t}}{W L C_{o x}^{2} f}
$$

where $\mathrm{C}_{\mathrm{ox}}$ is the gate dielectric capacitance per unit area, $\mathrm{q}$ is the electron charge, $k T$ is the thermal energy, $\lambda$ is the oxide tunneling attenuation distance, $\mathrm{N}_{\mathrm{t}}$ is the volumetric oxide trap density, WL is the channel area, and $f$ is frequency. On the other hand, the Hooge mobility fluctuations (HMF) model, which is more suitable for explaining the conduction mechanism in the volume of devices [16]-[19], suggests that $\mathrm{S}_{\mathrm{Id}} / \mathrm{I}_{\mathrm{d}}{ }^{2}$ is proportional to $1 / \mathrm{I}_{\mathrm{d}}[15]$. However, $\mathrm{S}_{\mathrm{Id}} / \mathrm{I}_{\mathrm{d}}{ }^{2}$ of the devices 

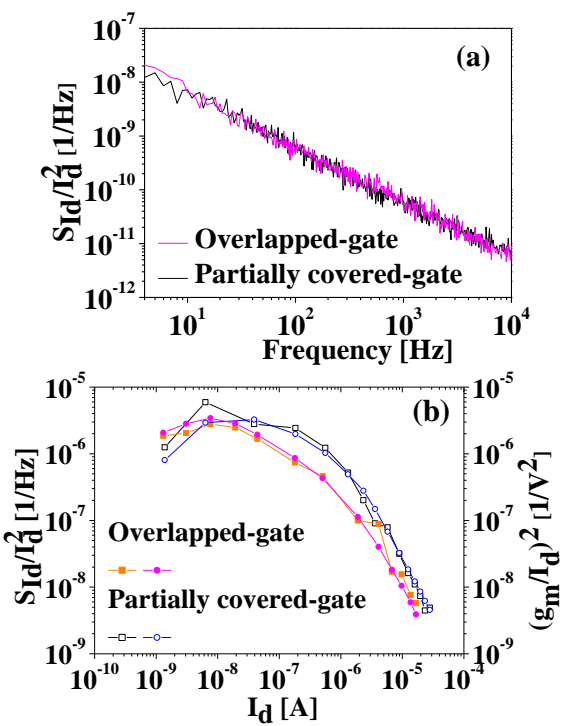

Fig. 3. (a) Normalized noise spectral density $\left(S_{\text {Id }}\right)$ as a function of frequency and (b) normalized $S_{\text {Id }}$ (left scale) matching with (constant $x$ $\left(g_{m} / l_{d}\right)^{2}$ ) (right scale) versus $I_{d}$ at $V_{d}=0.1 \mathrm{~V}$ and $f=10 \mathrm{~Hz}$ for the overlap and overlap-free $\mathrm{GaN}$ junctionless devices.

investigated in this work is well proportional to $\left(\mathrm{g}_{\mathrm{m}} / \mathrm{I}_{\mathrm{d}}\right)^{2}$, as shown in Fig. 3, which indicates that the noise of the GaN junctionless FinFETs is dominated by CNF rather than HMF, even though the channel in the fin body is separated from the $\mathrm{Al}_{2} \mathrm{O}_{3} / \mathrm{GaN}$ interface by the depletion region. This noise characteristic is consistent with the observed results in $\mathrm{Si}$ junctionless FinFETs [17]-[19]. In these works, it was shown that the diffusion through the depletion region or direct tunneling from the bulk neutral region to the interface results in the flat-band voltage fluctuations [18]-[19].

When matching $S_{\mathrm{Id}} / \mathrm{I}_{\mathrm{d}}{ }^{2}$ and $\left(\mathrm{g}_{\mathrm{m}} / \mathrm{I}_{\mathrm{d}}\right)^{2}$, the flatband voltage fluctuations $\left(\mathrm{S}_{\mathrm{vfb}}\right)$ are obtained to be $1 \times 10^{-8} \mathrm{~V}^{2} \cdot \mathrm{Hz}^{-1}$ and $4.5 \times$ $10^{-9} \quad \mathrm{~V}^{2} \cdot \mathrm{Hz}^{-1}$ for the partially covered- and the overlapped-devices, respectively. Considering the actual gate length for the overlapped-device is $\mathrm{L}_{\text {fin }}=2 \mu \mathrm{m}$, the corresponding trap density $\left(\mathrm{N}_{\mathrm{t}}\right)$ calculated using Equation (1) with $\lambda=0.11 \mathrm{~nm}$ is $2.1 \times 10^{20} \mathrm{~cm}^{-3} \cdot \mathrm{eV}^{-1}$ and $2.2 \times 10^{20}$ $\mathrm{cm}^{-3} \cdot \mathrm{eV}^{-1}$ [20], lower than the values reported from the GaN-based 3D devices [8]-[11]. This result suggests that the trap density of the GaN junctionless device is lower than the interface trap density in devices with surface channel.

Fig. 4 shows $\mathrm{S}_{\mathrm{Id}} \times f$ as a function of frequency at off-state $\left(\mathrm{V}_{\mathrm{g}}\right.$ $=-1 \mathrm{~V}$ ) for variable $\mathrm{V}_{\mathrm{d}}$ from $0.1 \mathrm{~V}$ to $5.0 \mathrm{~V}$. No spectral deformation is observed for the overlapped-device, which reflects that there is no generation-recombination (g-r) noise component because the noise well follows a $1 / f$ behavior, even though the active channel of the devices becomes fully depleted at off-state. However, for the partially covered-device, the product $\mathrm{S}_{\mathrm{Id}} \times f$ clearly presents two noise components: one is $1 / f$ and the other is g-r noise, described through the following equation [21].

$$
S_{I} f=K_{f}+\sum_{i=0}^{N} \frac{A_{i} f}{1+\left(\frac{f}{f_{o i}}\right)^{2}} \text { with } \tau_{i}=\frac{1}{2 \pi f_{o i}}
$$

where the first term explains the $1 / f$ noise component with coefficient of $\mathrm{K}_{f}$ and the second term is the sum of g-r noise components including the plateau value of $\mathrm{A}_{\mathrm{i}}$, the cutoff frequency $f_{\mathrm{oi}}$, and $\tau_{\mathrm{i}}$ is the trap time constant. $\mathrm{S}_{\mathrm{Id}} \times f$ for the
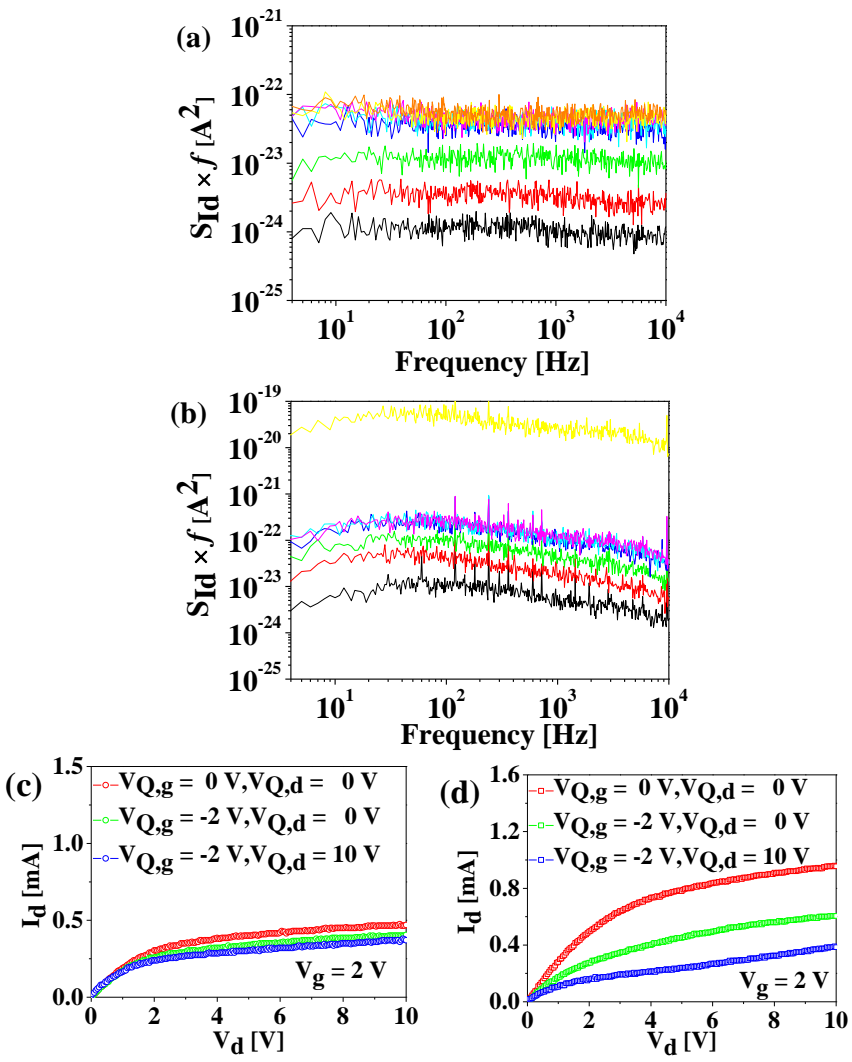

Fig. 4. Product $S_{I d} \times$ frequency versus frequency in the fabricated $G a N$ junctionless devices with (a) overlapped-and (b) partially covered-gate structure at $V_{g}=-1 \mathrm{~V}$ with $V_{d}$ varied from $0.1 \mathrm{~V}$ to $5 \mathrm{~V}$. Pulsed $I_{d}-V_{d}$ curves for the devices with (c) overlapped- and (d) partially covered-gate structure at $\mathrm{V}_{\mathrm{g}}=2 \mathrm{~V}$ sweeping $\mathrm{V}_{\mathrm{d}}=0 \sim 10 \mathrm{~V}$.

partially covered-device (Fig. 4(b)) dramatically decreases at approximately cutoff frequency $\left(f_{o i}\right)$ of $30 \sim 40 \mathrm{~Hz}$ due to the existence of g-r noise component, which indicates that the device has larger trap time constant in GaN buffer layer than that of the overlapped-device. In the overlapped-device buffer trapping is effectively reduced.

To further examine the buffer trapping effect observed from noise experiments, pulsed I-V measurements for both devices were conducted using Keysight B1500 semiconductor device analyzer with pulse width of $1 \mathrm{~ms}$, as shown in Fig. 4(c) and (d). The pulse conditions are three quasi-bias of (1) $\mathrm{V}_{\mathrm{g}, \mathrm{Q}}=\mathrm{V}_{\mathrm{d}, \mathrm{Q}}=0$ $\mathrm{V}$, (2) $\mathrm{V}_{\mathrm{g}, \mathrm{Q}}=-2 \mathrm{~V}, \mathrm{~V}_{\mathrm{d}, \mathrm{Q}}=0 \mathrm{~V}$, and (3) $\mathrm{V}_{\mathrm{g}, \mathrm{Q}}=-2 \mathrm{~V}, \mathrm{~V}_{\mathrm{d}, \mathrm{Q}}=10 \mathrm{~V}$. The drain voltage is swept from 0 to $10 \mathrm{~V}$ at $\mathrm{V}_{\mathrm{g}}=2 \mathrm{~V}$. The partially covered-device exhibits relatively large current collapse for all pulse conditions. The reason for this large current collapse is the wider effective trapping region, the ungated region between the gate and the drain electrode as shown in Fig. 1 [3], which includes the planar region between the gate and the drain, as well as the uncovered neutral fin array region which is not fully depleted. On the other hand, the overlapped-device shows much smaller current collapse, probably due to the reduced electric field at the gate edge caused by the overlapped gate structure along with smaller trapping region. The results obtained from the pulsed I-V characteristics of the GaN junctionless devices well match with the g-r noise performances at off-state. 


\section{REFERENCES}

[1] K.-S. Im, Y.-W. Jo, J.-H. Lee, S. Cristoloveanu, and J.-H. Lee, "Heterojunction-free GaN Nanochannel Finfets with High Performance," IEEE Electron Device Lett., vol. 34, no. 3, pp. 381-383, Mar. 2013. DOI: 10.1109/LED.2013.2240372

[2] K.-S. Im, C.-H. Won, Y.-W. Jo, J.-H. Lee, M. Bawedin, S. Cristoloveanu, and J.-H. Lee, "High-Performance GaN-Based Nanochannel FinFETs With/Without AlGaN/GAN Heterostructure", IEEE Trans. Electron Devices, vol. 60, no. 10, pp. 3012-3018, Oct. 2013. DOI: 10.1109/TED.2013.2274660

[3] K.-S. Im, D.-H. Son, H.-K. Ahn, S.-B. Bae, J.-K. Mun, E.-S. Nam, S. Cristoloveanu, and J.-H. Lee, "Performance improvement of normally off AlGaN/GaN FinFETs with fully gate-covered nanochannel", Solid-State Electron., vol. 89, pp. 124-127, Nov. 2013. DOI: 10.1016/j.sse.2013.08.001

[4] K.-S. Im, V. Sindhuri, Y.-W. Jo, D.-H. Son, J.-H. Lee, S. Cristoloveanu, and J.-H. Lee, "Fabrication of $\mathrm{AlGaN} / \mathrm{GaN} \Omega$-shaped nanowire fin-shaped FETs by a top-down approach", Appl. Phys. Express, vol. 8, no. 6, pp. 066501-1 3, Jun. 2015. DOI: 10.7567/APEX.8.066501

[5] K.-S. Im, C.-H. Won, S. Vodapally, R. Caulmilone, S. Cristoloveanu, Y. T. Kim, and J.-H. Lee, "Fabrication of Normally-Off GaN Nanowire Gate-All-Around FET with Top-Down Approach", Appl. Phys. Lett., vol. 109, no. 14, pp. 143106-1 4, Oct. 2016. DOI: 10.1063/1.4964268

[6] Y.-W. Jo, D.-H. Son, C.-H. Won, K.-S. Im, J. H. Seo, I. M. Kang, and J.-H. Lee, "AlGaN/GaN FinFET With Extremely Broad Transconductance by Side-Wall Wet Etch," IEEE Electron Device Lett., vol. 36, no. 10 , pp. 1008-1010, Oct. 2015. DOI: 10.1109/LED.2015.2466096

[7] A. L. McWhorter, "1/f Noise and Germanium Surface Properties in Semiconductor Surface Physics", Philadelphia, PA: Univ. Pennsylvania Press, 1957, pp. 207-208.

[8] S. Vodapally, Y. I. Jang, I. M. Kang, I.-T. Cho, J.-H. Lee, Y. Bae, G. Ghibaudo, S. Cristoloveanu, K.-S. Im, and J.-H. Lee, " $1 /$ f-noise in AlGaN/GaN nanowire omega-FinFETs," IEEE Electron Device Lett., vol. 38, no. 2, pp. 252-254, Feb. 2017. DOI: 10.1109/LED.2016.2645211

[9] S. Vodapally, C. G. Theodorou, Y. Bae, G. Ghibaudo, S. Cristoloveanu, K.-S. Im, and J.-H. Lee, "Comparison for $1 / \mathrm{f}$ Noise Characteristics of AlGaN/GaN FinFET and Planar MISHFET," IEEE Trans. Electron Devices, vol. 64, no. 9, pp. 3634-3638, Sep. 2017. DOI: 10. 1109/TED.2017.2730919

[10] J. H. Seo, Y. J. Yoon, D.-H. Son, J.-G. Kim, J.-H. Lee, J.-H. Lee, K.-S. Im, and I. M. Kang, "A Novel Analysis of Lgd Dependent-1/f noise in In0.08A10.92N/GaN," IEEE Electron Device Lett., vol. 39, no. 10, pp. 1552-1555, Oct. 2018. DOI: 10.1109/LED.2018.2865564

[11] K.-S. Im, M. S. P. Reddy, R. Caulmilone, C. G. Theodorou, G. Ghibaudo, S. Cristoloveanu, and J.-H. Lee, "Low-Frequency Noise Characteristics of GaN Nanowire Gate-All-Around Transistors With/Without 2DEG Channel", IEEE Trans. Electron Devices, vol. 66, no. 3, pp. 1243-1248, Mar. 2019. DOI: 10.1109/TED.2019.2894806

[12] K.-W. Kim, S.-D. Jung, D.-S. Kim, H.-S. Kang, K.-S. Im, J.-J. Oh, J.-B. Ha, J.-K. Shin, and J.-H. Lee, "Effects of TMAH Treatment on Device Performance of Normally Off A12O3/GaN MOSFET", IEEE Electron Device Lett., vol. 32, no. 10, pp. 1376-1378, Oct. 2011. DOI: 10.1109/LED.2011.2163293

[13] D. S. Lee, H. Wang, A. Hsu, M. Azize, O. Laboutin, Y. Cao, J. W. Johnson, E. Beam, A. Ketterson, M. L. Schuette, P. Saunier, and T. Palacios, "Nanowire Channel InAlN/GaN HEMTs With High Linearity of gm and fT," IEEE Electron Device Lett., vol. 34, no. 8, pp. 969-971, Aug. 2013. DOI: 10.1109/LED.2013.2261913

[14] J. A. Chroboczek and G. Piantino, France Patent No. 15075 (November 1999).

[15] G. Ghibaudo, O. Roux, C. Nguyen-duc, F. Balestra, and J. Brini, "Improved analysis of low frequency noise in field-effect MOS transistors," Phys. Status Solidi A, vol. 124, no. 2, pp. 571-581, 1991. DOI: $10.1002 /$ pssa.2211240225

[16] S. A. Hayat and B. K. Jones, "The excess noise in buried-channel MOS transistors," Semicond. Sci. Technol. vol. 2, no. 11, pp. 732-735, May. 1987. DOI: 10.1088/0268-1242/2/11/005

[17] K. Akarvardar, B. M. Dufrene, S. Cristoloveanu, P. Gentil, B. J. Blalock, and M. M. Mojarradi, "Low-Frequency noise in SOI Four-Gate Transistors," IEEE Trans. Electron Devices, vol. 53, no. 4, pp. 829-835, Apr. 2006. DOI: 10.1109/TED.2006.870272

[18] D. Jang, J. W. Lee, C.-W. Lee, J.-P. Colinge, L. Montès, J. I. Lee, G. T. Kim, and G. Ghibaudo, "Low-frequency noise in junctionless multigate transistors," Appl. Phys. Lett., vol. 98, no. 13, pp. 133502-1 3, Mar. 2011. DOI: $10.1063 / 1.3569724$

[19] D.-Y. Jeon, S. J. Park, M. Mouis, S. Barraud, G.-T. Kim, and G. Ghibaudo, "Low-frequency noise behavior of junctionless transistors compared to inversion-mode transistors," Solid-State Electron., vol. 81, pp. 101-104, Mar. 2013. DOI: 10.1016/j.sse.2012.12.003

[20] S. Sakong, S.-H. Lee, T. Rim, Y.-W. Jo, J.-H. Lee, and Y.-H. Jeong, "1/f Noise Characteristics of Surface-Treated Normally-Off Al2O3/GaN MOSFETs," IEEE Electron Device Lett., vol. 36, no. 3, pp. 229-231, Mar. 2015. DOI: 10.1109/LED.2015.2394373

[21] C. G. Theodorou, N. Fasarakis, T. Hoffman, T. Chiarella, G. Ghibaudo, and C. A. Dimitriadis, "Origin of the low-frequency noise in n-channel FinFETs," Solid-State Electron., vol. 82, pp. 21-24, Feb. 2013. DOI: 10. 1016/j.sse.2013.01.009

[22] M. Matys, K. Nishiguchi, B. Adamowicz, J. Kuzmik, and T. Hashizume, "Enhancement of channel electric field in $\mathrm{AlGaN} / \mathrm{GaN}$ multi-nanochannel high electron mobility transistors," J. Appl. Phys., vol. 124 , no. 22 , pp. $224502-1 \sim 8$, Nov. 2018. DOI: $10.1063 / 1.5056194$. 